\title{
Mit Abwechslung durchs Leben
}

\section{Adrian Ritter}

Freier Journalist

\begin{abstract}
Schwere Lasten heben ist selten, Bildschirmarbeit dafür für die meisten Arbeitnehmer Alltag geworden: Der technologische Wandel hat unsere Arbeitsplätze stark verändert. Der Arbeitsphysiologe Thomas Läubli hat diesen Wandel seit den 1970er Jahren erforscht. Die Frage, warum so viele Menschen unter Beschwerden des Bewegungsapparates leiden, wird den ETH-Wissenschaftler nach der Pensionierung weiter beschäftigen.
\end{abstract}

Eine solche Gelegenheit bietet sich nicht alle Tage: Als in den Schweizer Grossbanken um 1978 die ersten Bildschirm-Terminals installiert wurden, erhielt Thomas Läubli von seinem Doktorvater Etienne Grandjean einem der Begründer der Ergonomie - den Auftrag, die Auswirkungen der Bildschirmarbeit auf die Gesundheit der Angestellten zu erforschen. Einzelne Abteilungen in den zwei untersuchten Banken hatten die Terminals bereits eingeführt, andere noch nicht - eine seltene, ideale Voraussetzung für vergleichende Forschung. Die Resultate zeigten: Die Produktivität stieg dank der Bildschirmarbeit um den Faktor zehn, gleichzeitig nahmen bei den Angestellten aber Augenbeschwerden und Nackenschmerzen zu. Die Nackenschmerzen sollten Thomas Läubli als Forschungsobjekt sein ganzes Wissenschaftlerleben lang begleiten. Er untersuchte im Labor die Pathophysiologie

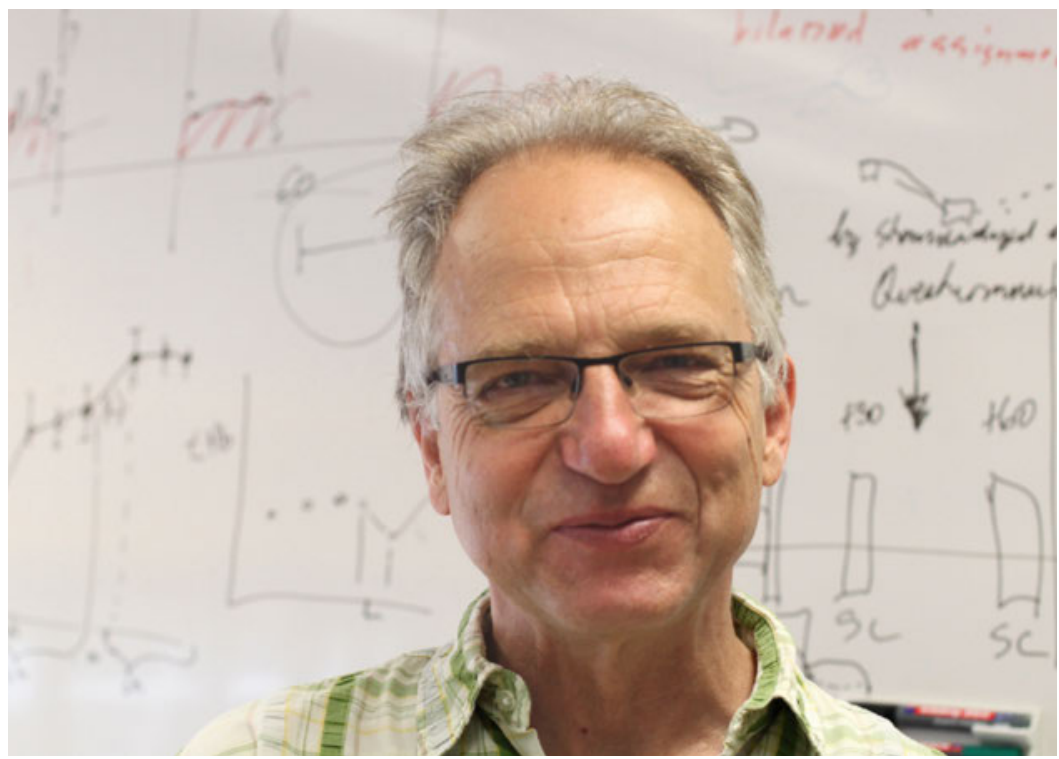

Abbildung 1: War in den 1980er Jahren allein auf weiter Flur mit der Aufteilung der Familien- und Berufsarbeit zwischen ihm und seiner Frau: Arbeitsphysiologe Thomas Läubli. von Nackenschmerzen, führte Studien zu Nackenschmerzen bei Pflegepersonal durch und ging dem Zusammenhang zwischen Nackenschmerzen und Schlafqualität nach. Neben den Nackenschmerzen widmete er sich in seiner Forschung einer grossen

Die Nackenschmerzen sollten Thomas Läubli als Forschungsobjekt sein ganzes Wissenschaftlerleben lang begleiten.

Bandbreite weiterer Themen - von der Wirkung von Lösungsmitteln auf das Zentralnervensystem bis zur Erhaltung der Leistungsfähigkeit bei Gerüstbauern.

\section{Ein junges Fachgebiet}

Eigentlich wollte Thomas Läubli Psychiater werden, als er 1978 das Medizinstudium an der Universität Zürich abschloss. Dann aber weckte ein Inserat des damaligen Instituts für Hygiene und Arbeitsphysiologie der ETH Zürich sein Interesse: Professor Etienne Grandjean suchte einen wissenschaftlichen Mitarbeiter. Läubli bewarb sich, erhielt die Stelle und liess sich vom Enthusiasmus der Kollegen im noch jungen Fachgebiet anstecken.

Seine Dissertation widmete er dem «arbeitsbedingten cervicobrachialen Überlastungssyndrom». Auch in der folgenden 40-jährigen wissenschaftlichen Tätigkeit lag der Schwerpunkt seiner Forschung bei muskulo-skelettalen Beschwerden im Zusammenhang mit der Arbeitstätigkeit. Nachdem die ETH Zürich das Institut für Hygiene und Arbeitsphysiologie 2004 geschlossen hatte, war Läubli am Zentrum für Organisations- und Arbeitswissenschaften derselben Hochschule tätig. Seit 2013 war er Privatdozent am Sensory-Motor Systems Lab der ETH Zürich, daneben seit 2008 Professor am Kyoto Institute of Technology. 


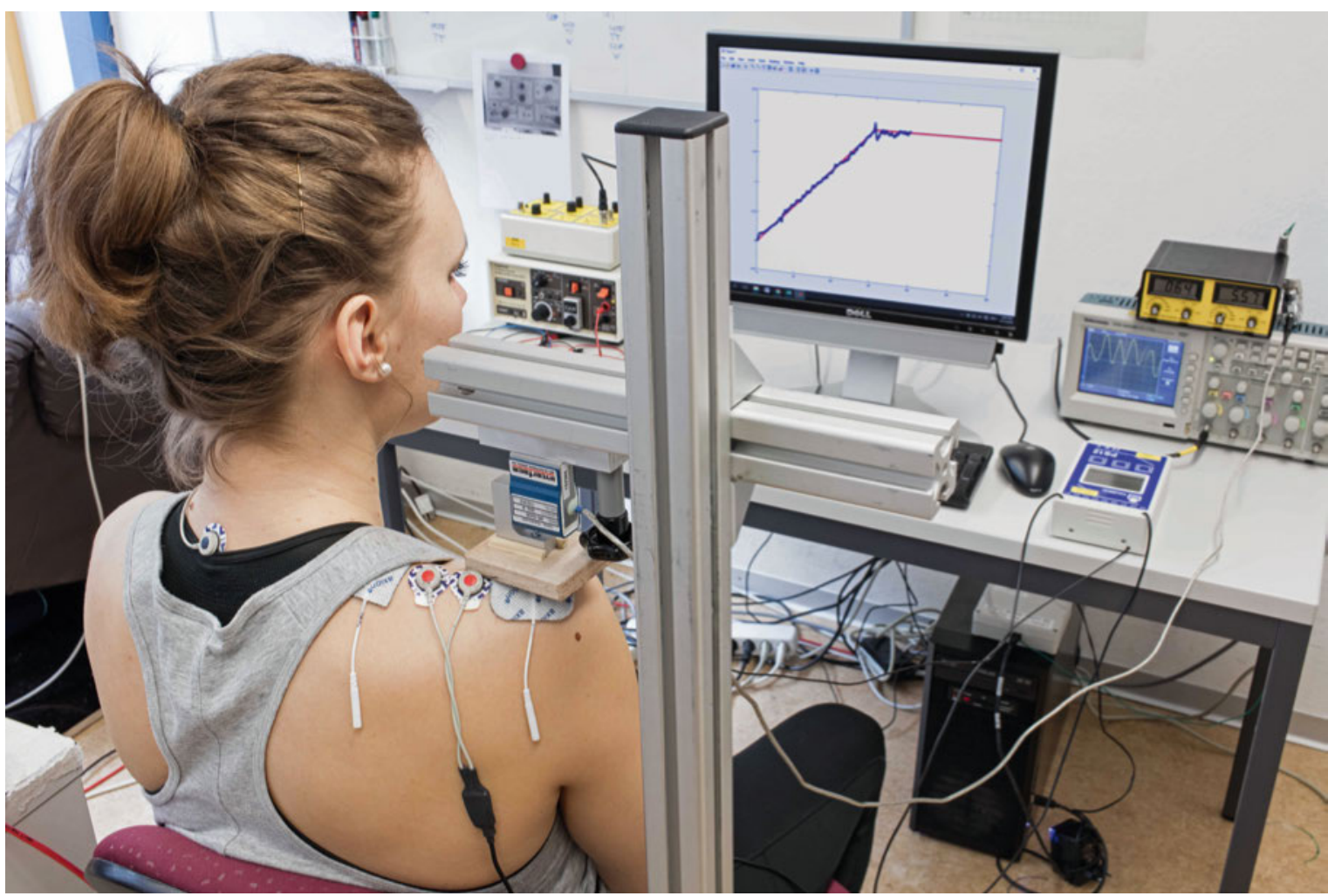

Abbildung 2: In einem seiner letzten Forschungsprojekte an der ETH Zürich hat Thomas Läubli mit seinem Team mittels Elektromyographie die Muskelaktivität während Computerarbeit gemessen.

\section{Von Prävention bis Therapie}

In seiner Forschung konnte er zeigen, dass Tastaturarbeit Beschwerden verursacht: je abgewinkelter die Stellung der Hände, desto ungünstiger. «Körperhaltungen verursachen grundsätzlich häufiger Beschwerden, wenn sie über längere Zeit eingenommen werden. Deshalb ist es wichtig, die Körperhaltung zu variieren", so Läubli. Er war auch einer der ersten Arbeitsphysiologen, die darauf hinwiesen, dass die Rückenlehne eines Stuhls nicht nur die Lende, sondern bis in den Schulterbereich hinauf stützen sollte.

\section{«Prävention und Gesundheitsförderung haben in der Politik heute einen schweren Stand."}

Nie war Thomas Läubli nur Forscher nach den Ursachen von Beschwerden, sondern immer auch an Prävention und Therapie interessiert. Er half mit, ergonomische Tastaturen zu entwickeln, schulte Mitarbeitende in Betrieben und entwickelte Rehabilitationsprogramme für verschiedene Berufsgruppen.

\section{«Allein auf weiter Flur»}

Langdauernde körperliche Beanspruchungen ohne genügend Pause gefährden die Gesundheit, diese Er- kenntnis zeigte Läubli in seiner Forschung immer wieder auf. Für ihn selber wurde die Forschung nie zu einer monotonen Tätigkeit. Dazu trug bei der Geburt seines Sohnes 1984 die Abmachung mit seiner damaligen Frau bei, zu gleichen Teilen für Kind und Haushalt zu sorgen. So war Thomas Läubli in den folgenden zwanzig Jahren zu 50 bis 60 Prozent berufstätig. In den 1980er Jahren war es Oberassistenten gerade erst gesetzlich ermöglicht worden, Teilzeit zu arbeiten. «Ich war allein auf weiter Flur mit dieser Aufteilung der Familien- und Berufsarbeit», sagt er rückblickend. Auch wenn seine Forschung in Teilzeit eine traditionelle wissenschaftliche Karriere verhindert haben mag - er bereut nichts: «Es war ein Glück, ein so vielseitiges Leben führen zu können. Es half mir auch, in der Forschung kreativ zu bleiben.»

\section{Der Wert der Prävention}

Arbeitsmedizinische Forschung ist interdisziplinäre Forschung. Je länger, je mehr spielten dabei für Thomas Läubli auch ökonomische Aspekte eine Rolle. In einer grossen Studie berechnete er 2014 die Gesundheitskosten hoher Arbeitsbelastungen in der Schweiz. Er kam allein für Rückenschmerzen auf mehr als 1,5 Milliarden Franken pro Jahr. Diese Kosten werden zum grössten Teil von der Allgemeinheit getragen - durch 
Krankenkassen und die Invalidenversicherung. Läubli kritisierte in der Studie, dass Arbeitgeber deshalb zu wenig Anreiz hätten, in den Gesundheitsschutz zu investieren. Er forderte, den Schutz vor arbeitsbedingten Erkrankungen zu stärken: "Präventionsmassnahmen könnten zu einer erheblichen Reduktion der IV-Kosten beitragen.»

Die Forderung aus der Studie wurde vom Parlament nicht aufgenommen. Läubli stellt im Gegenteil fest: «Prävention und Gesundheitsförderung haben in der Politik heute einen schweren Stand.» Jeder Arbeitnehmer sei selber für seine Gesundheit verantwortlich, sei dabei von bürgerlicher Seite oft zu hören. Nach zehn Jahren als Fachverantwortlicher «Ergonomie und arbeitsbezogene muskuloskelettale Erkrankungen" im Staatssekretariat für Wirtschaft (SECO) verliess Läubli deshalb 2016 die Behörde: "Ohne Rückhalt aus der Politik sah ich keine Perspektive mehr, etwas bewirken zu können.»

\section{Pausen machen, um nicht müde zu werden}

Arbeiten wir heute gesünder als früher, oder ungesünder? Weder noch, findet Thomas Läubli: «Die Belastungen haben sich verschoben.» Einerseits müssten heute zum Beispiel weniger Berufstätige schwere Lasten tragen - das werde von Maschinen übernommen. Andererseits arbeiteten die meisten Menschen heute inten-

\section{Arbeiten wir heute gesünder als früher, oder} ungesünder? Weder noch, findet Thomas Läubli: «Die Belastungen haben sich verschoben.»

siver - mit mehr Zeitdruck und Reizüberflutung, weniger Erholungszeit und häufig mit einer Vermischung von Arbeits- und Freizeit. «Die zunehmende Ökonomisierung prägt auch unsere Psyche. Die Menschen wollen immer produktiver werden - und pressen auch in ihre Freizeit immer mehr Aktivitäten. Die Anspannung nimmt zu», so Läubli. Er stellt fest, dass viele Menschen ein dysfunktionales Verständnis von Arbeit und Regeneration haben: «Für die meisten Menschen ist es normal, dass sie nach getaner Arbeit er- schöpft sind. Nur dann haben sie das Gefühl, etwas geleistet zu haben. Dem muss aber nicht so ein. Man kann auch unproduktiv müde werden", sagt Läubli. Im Vordergrund sollte aus seiner Sicht am Ende des Arbeitstages nicht das Müdigkeitsgefühl oder die Zahl der Arbeitsstunden stehen, sondern das effektiv Geleistete: «Man sollte nicht Pause machen müssen, um sich zu erholen, sondern um gar nicht erst müde zu werden.»

\section{Entzündung im Zentrum}

Ende Juli ging Thomas Läubli an der ETH Zürich in Pension. Ein Wermutstropfen bleibt: Seine Stelle wird nicht mehr besetzt, die Arbeitsphysiologie wird an den Hochschulen der Schweiz in Zukunft nur noch an einem Institut in Lausanne vertreten sein. Zu erforschen gäbe es aber noch viel, ist Läubli überzeugt. In einem 20-Prozent-Pensum am Institut für Arbeitsmedizin, Sozialmedizin und Versorgungsforschung der Universität Tübingen will er ein neues Konzept prüfen, das erklären soll, warum so viele Menschen unter Beschwerden am Bewegungsapparat leiden. Er ist überzeugt: «Die ständige Beanspruchung führt in den Muskeln, Sehnen, Bändern und Gelenken zu Entzündungen. Dieser Aspekt wird bisher zu wenig beachtet.»

Dass sein eigenes Leben auch in Zukunft nicht eintönig wird, dafür ist gesorgt. Neben seiner Arbeit spielt Läubli schon lange in einer Band Akkordeon und komponiert und arrangiert Stücke - das Repertoire reicht von Ländlermusik bis zu den Rolling Stones. Nach der Pensionierung will er sich zudem wieder mehr politisch engagieren. Das Thema Migration beschäftigt ihn: «Abgewiesene Asylsuchende, die aus völkerrechtlichen Gründen Schutz in der Schweiz erhalten, müssen oft über viele Jahre in Zivilschutzunterkünften ohne Tageslicht leben und dürfen nicht arbeiten. Das ist inakzeptabel. Kein Wunder, werden viele zusätzlich zum schon Erlebten krank davon. Zu viel Arbeit ist schädlich, zu wenig auch.»

\section{Bildnachweise}

Abb. 1: Bild: Adrian Ritter

Abb. 2: $\mathrm{zVg}$ 